\title{
A Review of the Literature on Culture-Negative Periprosthetic Joint Infection: Epidemiology, Diagnosis and Treatment
}

\author{
Hong-Kwon Yoon, $\mathrm{MD}^{1}$, Seong-Hee Cho, $\mathrm{MD}^{1}$, Dong-Yeong Lee, $\mathrm{MD}^{1}$, Byeong-Hun Kang, $\mathrm{MD}^{1}$, \\ Sang-Hyuk Lee, $\mathrm{MD}^{2}$, Dong-Gyu Moon, $\mathrm{MD}^{3}$, Dong-Hee Kim, $\mathrm{MD}^{1}$, Dae-Cheol Nam, $\mathrm{MD}^{1}$, and \\ Sun-Chul Hwang, $\mathrm{MD}^{1}$ \\ ${ }^{1}$ Department of Orthopaedic Surgery, Gyeongsang National University Hospital, Gyeongsang National University School of Medicine, Jinju; ${ }^{2}$ Department of \\ Orthopaedic Surgery, Gangneung Armed Hospital, Gangneung; ${ }^{3}$ Department of Orthopaedic Surgery, Gyeongsang National University Changwon Hospital, \\ Changwon, Korea
}

\begin{abstract}
Purpose: The prevalence of periprosthetic joint infection (PJI) has increased with the increasing incidence of arthroplasty surgery. Considering identification of causative microorganisms is crucial for treatment of PJI, culture-negative (CN) PJI is a significant clinical issue. The purpose of the present study is to describe epidemiology, diagnosis and treatment of CN PJI based on review of the literature to help prevent delayed diagnosis and improve clinical outcomes of CN PJI.

Methods: MEDLINE, EMBASE, Cochrane Library and Scopus databases were searched for articles on CN PJI. Only clinical studies written in English were included. Basic science studies, letters to the editor, case reports and review articles on PJI were excluded.

Results: Seven studies were included in this study. The prevalence of CN PJI ranged from $0 \%$ to $42.1 \%$. The major risk factors for CN PJI were prior antibiotic use and presence of postoperative wound drainage. Vancomycin and cephalosporins were the most commonly used antibiotics for CN PJI. Two-stage revision arthroplasty followed by 6 weeks of antibiotic therapy produced the most successful treatment outcomes.

Conclusions: In most clinical studies on CN PJI, a definite diagnostic method for identifying causative microorganisms or optimal treatment strategy for CN PJI were not clearly described. Therefore, further studies are needed to establish standard diagnostic methods for identifying infecting organisms and treatment strategies for CN PJI.
\end{abstract}

Keywords: Arthroplasty, Infection, Culture, Review

\section{Introduction}

Prosthetic joint arthroplasty is one of the most commonly per-

Received June 15, 2016; Revised (1st) August 17, 2016;

(2nd) October 8, 2016; (3rd) November 3, 2016;

Accepted November 8, 2016

Correspondence to: Sun-Chul Hwang, MD

Department of Orthopaedic Surgery, Gyeongsang National University

Hospital, Gyeongsang National University School of Medicine, 79

Gangnam-ro, Jinju 52727, Korea

Tel: +82-55-750-8102, Fax: +82-55-761-9477

E-mail: hscspine@hanmail.net

This is an Open Access article distributed under the terms of the Creative Commons Attribution Non-Commercial License (http://creativecommons.org/licenses/by-nc/4.0/) which permits unrestricted non-commercial use, distribution, and reproduction in any medium, provided the original work is properly cited. formed surgical procedures in the field of orthopedics. Among many complications of prosthetic joint arthroplasty, periprosthetic joint infection (PJI) is the most catastrophic complication ${ }^{1)}$. The prevalence of PJI appears to be on the rise with the increasing incidence of arthroplasty surgery. However, management of PJI is challenging due to the lack of standardized guidelines, especially in the case of culture-negative (CN) PJI.

The prevalence of CN PJI has been reported to range between $0 \%$ and $42.1 \%^{2-5)}$. Identification of microorganisms causing PJI is a critical task for selection of appropriate treatment options and prognosis prediction; therefore, CN PJI is considered an important clinical issue. Some studies have demonstrated that there are no significant differences in clinical characteristics between culture-positive (CP) PJI and CN PJI ${ }^{6,7)}$. In addition, distinctive clinical characteristics of CN PJI have not been well established. 
Therefore, diagnosis of CN PJI is difficult and often delayed. Diagnosis of PJI depends on the medical history and physical examination in combination with serologic tests and radiographic evaluation. However, clinical manifestations and serologic tests are not always reliable, and preoperative inflammatory indexes such as erythrocyte sedimentation rate (ESR), C-reactive protein (CRP) in serum and cell count in synovial fluid have limitations. Isolation of an organism from preoperative joint fluid aspiration, intraoperative culture or tissue biopsy is most useful for the selection of appropriate antibiotics and prediction of treatment responses. Furthermore, when the diagnosis of PJI is unclear, it is important to determine whether the culture outcome is true-negative or false-negative due to the presence of rare microorganisms such as mycobacteria or fungi. Based on the outcome, the former can be diagnosed as aseptic loosening and the latter as CN PJI, and different treatment options should be explored.

An accurate diagnosis of PJI helps patients avoid unnecessary multiple surgical procedures and inappropriate treatments involving prolonged use of parenteral antibiotics. The proper treatment of PJI remains controversial and depends on various factors such as presentation of symptoms, comorbidities, prosthetic factors, pharmacological factors and pathogenic factors ${ }^{8}$. However, in the case of CN PJI, choosing an appropriate antibiotic therapy is especially difficult. Furthermore, the effectiveness of a local intra-articular antimicrobial spacer in two-stage arthroplasty is unclear. Therefore, for successful treatment of CN PJI, systematic understanding of the disease entities of CN PJI is of utmost importance.

The aim of the present study was to systematically review previous clinical studies on CN PJI, evaluate epidemiology, diagnosis and treatment options with an intention to understand the disease entities for successful infection control. Therefore, a descriptive analysis of clinical data on CN PJI documented in the literature was conducted for this review article.

\section{Methods}

A systematic review was undertaken on clinical studies. A systematic electronic search was performed using the MEDLINE via PubMed and EMBASE, the Cochrane Library and Scopus databases. The search was carried out in January 2016 in the presence of two observers. The following key search terms were used in all fields: "arthroplasty", "knee prosthesis", "hip prosthesis", "infection", and "culture". The search was restricted to English language publications regardless of the year of publication. The complete search strings are shown in the Appendix 1. Unpublished data, case reports, letters to editor, instructional courses and review articles were not included. Two of the authors independently screened the titles and abstracts from the search results, and in the case the abstract was not found to be sufficient, the full-text was reviewed to evaluate the suitability of inclusion. Any disagreement between the two observers was resolved by consensus following discussion. Finally, the reference lists of the selected studies were investigated for the purpose of identification of additional studies that were not found through the electronic search.

The subject of studies included in the present study was PJI after arthroplasty of the hip and knee. Arthroplasties of the shoulder, ankle and hand were not included due to the rarity of studies with respect to PJI. Considering the existence of certain controversies with respect to the definition of PJI, we chose to include all papers in which the authors stated that the diagnostic criteria of PJI they applied were consistent with those of the Musculoskeletal Infection Society (MSIS) $)^{8)}$. The MSIS criteria are as follows: 1 ) there is a sinus tract communicating with the prosthesis; 2) a pathogen is isolated by culture from 2 or more separate tissue or fluid samples obtained from the affected prosthetic joints; or 3) four of the following 6 conditions are observed: a) elevated serum ESR and CRP concentration, b) elevated synovial white blood cell count, c) elevated synovial polymorphonuclear (PMN) percentage, d) presence of purulence in the affected joint, e) isolation of a microorganism in one culture of periprosthetic tissue or fluid, or f) greater than 5 neutrophils per high-power field in 5 highpower fields observed from histologic analysis of periprosthetic tissue at $\times 400$ magnification. In other words, all studies in the present study had sufficient laboratory results to meet the MSIS criteria for PJI. Furthermore, treatment failure was also defined as follows: 1) $\geq 2$ cultures positive for the same organism, 2) the presence of acute inflammation on pathologic findings, 3) development of a sinus tract, 4) presence of purulence in the joint space or around the arthroplasty site, or 5) clinical, radiological, and laboratory findings suggestive of $\mathrm{PJI}^{2}$.

For the purpose of comparative analysis of the outcomes of each study, a data sheet containing the author's name, year of publication, study design, number of cases, prevalence of PJI, risk factors, diagnosis, two-stage exchange arthroplasty success rate, used antibiotics and clinical outcomes was designed. The use of irrigation and debridement as an appropriate alternative for treatment of PJI still remains as a controversial issue. One-stage exchange arthroplasty has been performed for PJI because of certain advantages over two-stage exchange; however, the treatment success rate of one-stage exchange arthroplasty for PJI has been reported to be lower than that of two-stage exchange arthroplasty. 


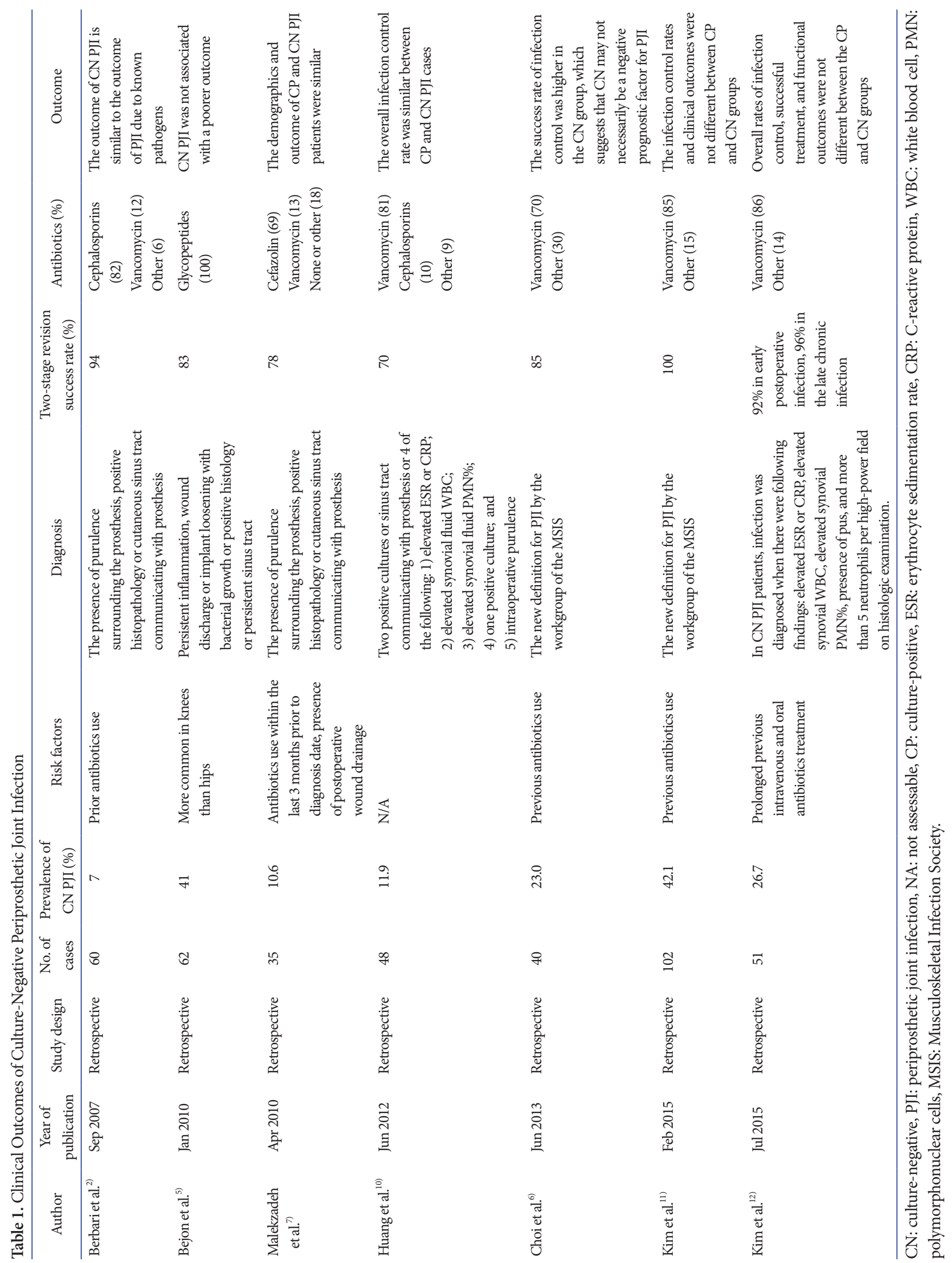


Although there are several different surgical techniques for PJI, two-stage exchange arthroplasty is the most widely performed procedure, producing superior clinical outcomes compared to other surgical techniques ${ }^{9}$. For these reasons, only the treatment success rate of two-stage exchange arthroplasty presented in the data sheet. The data were extracted from the included studies according to a predefined standardized data sheet.

A descriptive review of epidemiology, diagnosis, treatment and clinical outcome documented in the included studies on PJI was performed, and comparisons with other studies was carried out (Table 1). In cases of disagreement, consensus was reached through discussion.

\section{Results}

There were 339 search results obtained from MEDLINE via PubMed, 644 from EMBASE, 2 from the Cochrane Library and 390 from Scopus using the previously described search terms. From a total of 1,375 studies, 1,323 were excluded based on the respective titles and abstracts that did not meet the inclusion criteria of the present work. From the remaining 52 papers, seven were selected by the two observers and the remaining 45 papers were considered for further discussion and excluded by consensus. Consequently, seven papers were selected for final inclusion in the systematic review (Fig. 1). The review results of 7 included studies are summarized in Table $1^{2,5-7,10-12)}$. The other 45 studies were mostly excluded because they discussed either only diagnosis or treatment plans for CN PJI or did not focus on CN PJI.

All papers included in the present study were published between September 2007 and July 2015. The papers had six different first authors, were written in English and were published in six different journals including Clinical Infection Diseases $(\mathrm{n}=1)$, Journal of Antimicrobial Chemotherapy ( $\mathrm{n}=1$ ), Clinical Orthopae- dics and Related Research $(\mathrm{n}=2)$, The Journal of Arthroplasty $(\mathrm{n}=1)$, Journal of Orthopaedics $(\mathrm{n}=1)$ and Archives of Orthopaedic and Trauma Surgery $(\mathrm{n}=1)$. All the included studies were of retrospective design and the total number of CN PJI cases was 498 .

In every individual study, the incidence of CN PJI was reported to range from $7.0 \%$ to $42.1 \%$ and most of the commonly used antibiotics belonged to the group of glycopeptides such as vancomycin. Risk factors for CN PJI documented in the included studies included prior antibiotic use and presence of postoperative wound drainage. Prior antibiotic use was associated with circumstances where an antibiotic was empirically used under suspicion of diagnosis of local infection before confirmation of the diagnosis of PJI or culture test or a long-term use of antibiotics after surgery in presence of postoperative wound drainage or during the postoperative follow-up; however, duration of administration was not confirmed due to insufficient data. The most common surgical intervention was two-stage exchange arthroplasty. First, the authors performed debridement and removal of all prosthetic components, and subsequently an antibiotics (vancomycin and gentamycin impregnated)-mixed mobile cement spacer was inserted. Then, antibiotics were administered intravenously for a period of six weeks or more. If laboratory test results showed no evidence of infection, they performed reimplantation as a second stage of treatment. Other reported surgical options for $\mathrm{CN}$ PJI included debridement and retention of the prosthesis with replacement of the polyethylene insert of the tibial component, one-stage exchange of prosthesis, permanent resection arthroplasty without reimplantation and others. Despite the presence of various surgical options, this systematic review revealed that two-stage exchange arthroplasty is the most successful surgical treatment strategy for CN PJI. Almost all included studies demonstrated that the treatment success rate of CN PJI is similar to that of CP PJI. However, only one of the studies suggested that
Records identified through database searching MEDLINE $(n=339)$, EMBASE $(n=644)$, Cochrane Library $(n=2)$, Scopus $(n=390)$, Total $(n=1,375)$

\begin{tabular}{|c|c|}
\hline & $\begin{array}{l}\text { Records excluded based on } \\
\text { the title and the abstract } \\
\qquad(n=1,323)\end{array}$ \\
\hline $\begin{array}{c}\downarrow \\
\begin{array}{c}\text { Full-text articles assessed } \\
\text { for eligibility }(n=52)\end{array}\end{array}$ & \multirow{2}{*}{$\begin{array}{l}\text { Reasons for exclusion }(n=45) \\
\text { - Study focusing on only diagnosis or treatment for PJI } \\
\text { - Not written in English } \\
\text { - Basic science study or case report } \\
\text { - Review article }\end{array}$} \\
\hline & \\
\hline
\end{tabular}

Studies included in systematic review $(n=7)$
Fig. 1. PRISMA (Preferred Reporting Items for Systematic Reviews and Meta-analyses) flow diagram. PJI: periprosthetic joint infection. 


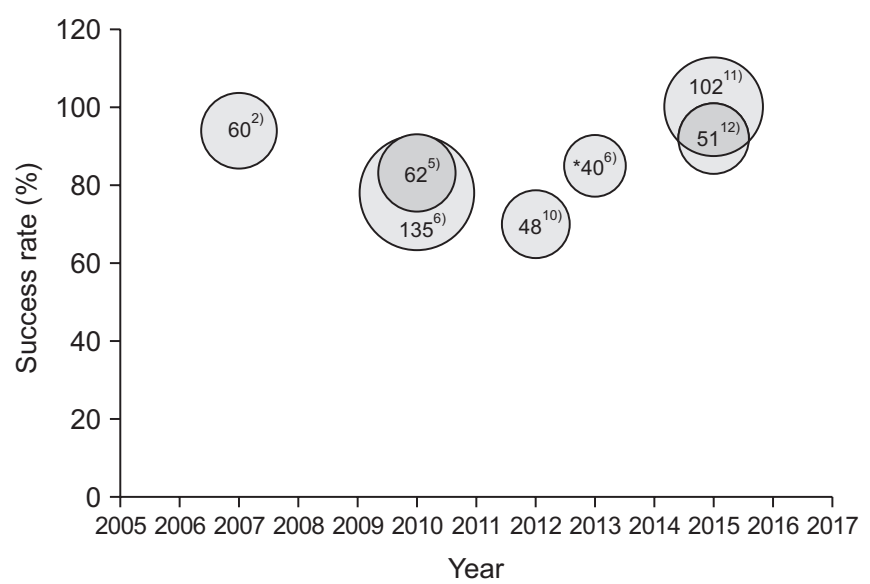

Fig. 2. Success rates of two-stage revision arthroplasty of the included studies ${ }^{2,-7,10-12)}$.

${ }^{*}$ A higher success rate of infection control in the culture-negative group was reported only in this study.

although there were no significant differences in clinical characteristics between $\mathrm{CN}$ and $\mathrm{CP}$ groups, the success rate of infection control was higher in the $\mathrm{CN}$ group, which suggests that culture negativity may not necessarily be a negative prognostic factor for PJI (Fig. 2) 2,5-7,10-12).

\section{Discussion}

Growing interest in CN PJI has led to an increase in the number of clinical research evaluating difficulties confronted during diagnosis and treatment and clinical outcomes. However, there is a paucity of literature on definite diagnostic methods and optimal treatment methods for CN PJI. Under such circumstances, it is difficult to determine the most appropriate clinical setting for the treatment of CN PJI.

$\mathrm{PJI}$ is one of the most challenging complications after total hip arthroplasty (THA) and total knee arthroplasty (TKA). It has been reported that the incidence of PJI ranges from $1 \%$ to $4 \%$ after primary TKA and $1 \%$ to $2 \%$ after primary THA ${ }^{1,13)}$. However, despite extensive efforts for the diagnosis of PJI based on identification of causative microorganisms and clinical symptoms, clinical suspicion, radiological evaluation and laboratory tests (ESR, CRP, PMN percentage), culture outcomes often reveal high false-negative rates and the incidence of negative cultures in most infection series has been reported to range from $0 \%$ to $42.1 \%^{2-5}$. Thus, it is imperative to have thorough knowledge about the infecting microorganism. A failure to identify the infecting microorganism leads to inappropriate treatment with a subsequent increase in treatment failure ${ }^{14)}$. The detection of CN PJI was based on the results of inaccurate and inappropriate diagnostic tools for rare organisms such as fungi, mycobacterium, and fastidious bacteria that are not easily detected through routine methods ${ }^{2,7}$. Some studies reported that $46 \%$ of CN PJI were caused by fungi, $43 \%$ by mycobacteria and $11 \%$ by other bacteria such as Listeria monocytogens, Propionibacterium acnes, Brucella, Coxiella burnetii and others ${ }^{2,7)}$. In addition, Million et al. ${ }^{15)}$ reported that $35 \%$ of CN PJI were caused by Brucella and 16\% were caused by Coxiella burnetii, excluding all cases of fungi and mycobacteria. Therefore, in cases where negative culture is the outcome, the likelihood of presence of these rare organisms needs to be considered for appropriate treatment planning. Also, in order to detect such low virulent organisms, acquisition of multiple samples and sufficient growth time are required.

The diagnosis of CN PJI is a challenging task. In the absence of accurate diagnostic methods, clinicians rely on physical examination, clinical suspicion, laboratory tests, and radiological findings. However, clinical symptoms and signs are not always reliable and currently available laboratory tests using synovial fluid have limitations. Furthermore, preoperative radiographic findings are only useful for the diagnosis of chronic stage CN PJI. Thus, there has been growing interest in diagnostic methods to improve treatment success rates of CN PJI. Among various diagnostic tools for CN PJI, laboratory tests are most critical for appropriate identification of the infecting microorganism and treatment strategy planning. However, prior antibiotic use can compromise the sensitivity of traditional diagnostic laboratory tests. Berbari et al. ${ }^{2)}$ reported in their study that $53 \%$ of patients with CN PJI had received antibiotic therapy before culture samples were obtained from the affected joint. To increase the sensitivity of traditional diagnostic tests, the clinical practice guideline of the American Academy of Orthopaedic Surgeons recommends withholding antimicrobials for at least two weeks before aspiration of the joint ${ }^{16)}$. Furthermore, in an attempt to improve the sensitivity or specificity for the diagnosis of CN PJI and reduce the number of false-negative results, recent studies have focused on basic molecular biology techniques ${ }^{17}$. The most common molecular biology techniques that have been used to diagnose PJI are based on polymerase chain reaction (PCR). Melendez et al. ${ }^{18)}$ proved PCR to be valuable for detecting microorganisms in synovial fluid in $88 \%$ of cases, and demonstrated that PCR can be used to detect unusual species such as Candida and antibiotic-resistant methicillin-resistant Staphylococcus aureus (MRSA). In the study, they suggested that the use of PCR with mass spectrometry was shown to identify four out of five $\mathrm{CN}$ cases. Bereza et al. ${ }^{19)} \mathrm{dem}$ onstrated bacterial DNA isolation using PCR in $90 \%$ of patients 
with CN synovial fluid. However, PCR is extremely sensitive whereas specificity is low; therefore, it is inappropriate to be used as a single diagnostic tool. Another test for the diagnosis of PJI is to evaluate alpha-defensin ${ }^{17}$. The alpha-defensin test has shown promising results for diagnosing PJI, and the overall sensitivity and specificity of the alpha-defensin test is greater than $95 \%{ }^{20,21)}$. Prior use of antibiotics for infection control decreases the level of laboratory markers such as ESR, CRP and PMN percentage. However, the alpha-defensin test maintains its concentration for PJI even in the case of antibiotic administration without any impact on its level of sensitivity ${ }^{17,20)}$. For these reasons, the alphadefensin test is thought to provide excellent utility as a screening test for PJI. Other interesting diagnostic methods for CN PJI have been introduced such as sonication with $\mathrm{PCR}^{22)}$. Ibs T5000 biosensor (Ibis Biosciences Inc., Carlsbad, CA, USA) ${ }^{23)}$ and PLEXID (Abbott Laboratories, Abbott Park, IL, USA) ${ }^{24}$. However, for clinical orthopedic surgeons, because of the limitations of technical availability, there arises a need for commercial development of such devices. Thus, for the treatment of PJI, confirmation of the pathogen is crucial for determination of treatment strategy or use of antibiotics, and it is important to develop easily applicable diagnostic methods.

Antibiotic use in addition to surgical treatment is necessary for effective elimination of an infection in PJI. Henceforth, it is important for the clinicians to identify cultured microorganisms for selection of sensitive antibiotics and determination of parenteral antibiotic therapy after consultation with an infectious disease specialist. To date, a consensus has not yet been established concerning standardized criteria on the initial use of antibiotics for $\mathrm{CN}$ PJI. If an infecting microorganism is preoperatively identified, prophylactic preoperative antibiotics should be administered. However, even if identification of an infecting microorganism is not achieved, in the absence of conclusive evidence on antibiotic holiday periods, a few authors have suggested that continuous use of antibiotics and prophylactic antibiotics needs to last for at least 2 weeks ${ }^{7,25}$. Accordingly, an increase in the sensitivity to detect infecting microorganisms in intraoperative cultures can be achieved. In the present study, 5 of the 7 studies extensively employed glycopeptides such as vancomycin and the other two studies more commonly used cephalosporins. Among 498 cases, approximately 284 cases used glycopeptides such as vancomycin and the other 214 cases used cephalosporins, betalactam, quinolones or a combination therapy for treating CN PJI. Since PJIs are frequently caused by either Staphylococcus aureus or coagulase-negative Staphylococcus species, numerous researchers are especially using cephalosporins as a medical treatment for
$\mathrm{CN} \mathrm{PJI}{ }^{2,6)}$. However, due to the increased failure rates associated with MRSA infection, vancomycin administration has been suggested as an alternative treatment ${ }^{26)}$. Cuckler ${ }^{27)}$ reported that no significant association was found between the empirical parenteral vancomycin therapy and treatment failure in patients with CN PJI. In addition, Choi et al. ${ }^{6}$ also reported a high-dosage vancomycin appeared to have contributed to favorable outcomes in patients with CN PJI. The increased use of vancomycin has been associated with the growing incidence of MRSA infection, and infectious disease specialists recommend dual antibiotic therapy using vancomycin with ceftriaxone or a fluoroquinonlone. However, the routine use of vancomycin predisposes patients to acute kidney injury ${ }^{28)}$, and there is a lack of evidence that the addition of vancomycin as a prophylactic antibiotic agent is more effective in decreasing the incidence of infection than the use of cefazoline alone ${ }^{29)}$; therefore, further studies are required to determine the superiority of either the single and dual use of prophylactic antibiotics. Apart from the nature of antibiotics, the duration of antibiotic therapy also plays a significant role in favorable results. As per the literature review, current recommendations for $\mathrm{CP}$ PJI include 4 weeks to 6 months of antibiotic therapy based on the microorganism identified ${ }^{5,30)}$. Parenteral antibiotics is mainly used for 6 weeks for CN PJI; however, the appropriate duration of oral antibiotic therapy for CN PJI followed by surgical treatment has yet to be established. CN PJIs are often the cause of anxiety for both the patient and the surgeon under circumstances when diagnosis is difficult due to failure to identify the infecting microorganism $^{5,11,12)}$. In addition, a long-term use of broad-spectrum antibiotics or multiple antibiotics against the most common infecting organisms can have a risk of toxicity and may not provide coverage against fungi or rare organisms ${ }^{31}$. Therefore, a successful antibiotic treatment strategy for CN PJI requires accurate identification of antibiotics and correct diagnosis.

Regardless of culture results, it is clear that the choice of surgical option greatly affects the treatment results of PJI. Surgical treatment methods for CN PJI include debridement and retention of implant, one-stage exchange arthroplasty, and two-stage exchange arthroplasty. For early infections occurring within three postoperative weeks and acute-onset hematogenous infections with less than three weeks of symptoms, debridement and retention of implant and antibiotic therapy are performed. In general, debridement should be open arthrotomy rather than arthroscopy, and the polyethylene liner should be changed ${ }^{32}$. This surgical option is less invasive and less complex and costs less than exchange revision surgery ${ }^{33)}$. Hartman et al. ${ }^{34)}$ reported significant improvement in the success rate of debridement and retention of implant 
when performed within four weeks of index knee surgery. Furthermore, Koyonos et al. ${ }^{35}$ ) suggested that successful treatment outcomes of debridement and retention of implant were significantly associated not only with the short duration of symptoms but also with the presence of comorbidities and infections caused by microorganisms of lower virulence. However, there still exists certain controversies regarding its efficacy. Brandt et al ${ }^{36)}$ reported that the procedure resulted in poor outcomes in PJI patients: infection control was achieved in less than $40 \%$. Kim et al. ${ }^{11)}$ also reported unsatisfactory outcomes: the treatment success rate was $62 \%$ for early deep infection and $25 \%$ for acute hematogenous infection. Because of such reports showing unsatisfactory outcomes of debridement and retention of implant, prosthesis exchange is currently recommended. The infection control rate of one-stage revision surgery has been reported to range from $73 \%$ to $93 \%$ in previous studies; however, none of the seven included studies recommended one-stage revision as the first treatment option for CN PJI, and they reported that the success rate of two-stage exchange arthroplasty was superior to one-stage exchange arthroplasty ${ }^{2,5-7,10-12)}$. Most authors of the included studies reported that two-stage exchange arthroplasty followed by 4-6 weeks of antibiotic therapy was effective in all stages of CN PJI with the success rate ranging from $70 \%$ to $100 \%$. A recent systematic review also reported the average success rate of two-stage exchange arthroplasty for PJI as $90 \%$. The study also reported that twostage exchange arthroplasty provided better outcomes than onestage exchange arthroplasty for $\mathrm{PJI}^{37)}$. Therefore, it is our understanding that among various surgical treatment methods for $\mathrm{CN}$ PJI, debridement and retention of implant should be considered as an option in limited cases including early infections (within 3 weeks), in cases where the patient is in good general medical condition, or in the presence of infections caused by microorganisms of lower virulence. Patients with late chronic PJI or an infection caused by highly virulent microorganisms should be considered for two-stage exchange arthroplasty. Furthermore, in cases where an implant exchange surgery fails to improve mobility or joint function, permanent removal of the prosthesis or amputation should be considered.

Although the clinical treatment outcomes of PJI with confirmed infecting microorganisms have been well documented, few reports exist on clinical outcomes and demographic characteristics of patients with CN PJI. In case of failure to isolate infected microorganisms and initiation of empirical antibiotic therapy without identification of microorganisms, there can be high chances of treatment failure resulting in poor clinical outcomes. However, in six out of seven papers included in the pres- ent study, the clinical outcomes and infection control rates were not different between CP PJI and CN PJI groups. Among seven studies, Choi et al. ${ }^{6}$ reported a higher infection control rate in the CN PJI group. These results mean that culture negativity may not necessarily be a negative prognostic factor for PJI. Conversely, a study by Mortazavi et al. ${ }^{38}$, which is not included in the present work, suggested that the PJI cases in which the microorganisms could not be isolated (CN PJI) had a four-fold increased risk of failure. In summary, there were no significant differences in clinical outcomes and infection control rates between CP PJI groups and CN PJI groups in previous studies based on our review; however there are some controversial questions that need to be answered considering the presence of studies reporting results not congruent with ours. The different treatment outcomes may be associated with differences in the antibiotic therapy, surgeons who performed the revision procedure and demographic factors, all of which need to be addressed in massive prospective studies with independent factors under control.

There are several limitations of the present systematic review. First, this study included PJI after THA in addition to TKA; however, we believe this could contribute to comprehensive understanding of PJI by not limiting specifically to PJI in the knee joint. Second, this review included only CN PJI studies of retrospective design. This was because randomized controlled trials on clinical results of CN PJI could not be traced through various databases. Third, the search was limited to papers written in English on MEDLINE via PubMed, EMBASE, the Cochrane Library and Scopus. Finally, studies included in this review adopted definition of PJI devised by MSIS. However, there is a growing tendency of using the definition of PJI provided by the International Consensus Group ${ }^{39,40)}$, and future studies may reckon such tendency. To overcome these limitations, we think that more prospective research on PJI should be encouraged and studies written in languages besides English should also be thoroughly reviewed. Despite these limitations, the significance of this systematic review is that it is a rare study addressing overall factors associated with $\mathrm{CN}$ PJI to contribute to identification of various factors that affect clinical outcome and optimal treatment strategies for CN PJI.

In most clinical studies on CN PJI, definite diagnostic methods for isolation of infecting microorganisms or optimal treatment strategies for CN PJI were not clearly described. Therefore, further prospective studies need to be performed to establish the standard diagnostic methods and optimal treatment strategies for CN PJI. 


\section{Conflict of Interest}

No potential conflict of interest relevant to this article was reported.

\section{References}

1. Phillips JE, Crane TP, Noy M, Elliott TS, Grimer RJ. The incidence of deep prosthetic infections in a specialist orthopaedic hospital: a 15-year prospective survey. J Bone Joint Surg Br. 2006;88:943-8.

2. Berbari EF, Marculescu C, Sia I, Lahr BD, Hanssen AD, Steckelberg JM, Gullerud R, Osmon DR. Culture-negative prosthetic joint infection. Clin Infect Dis. 2007;45:1113-9.

3. Parvizi J, Ghanem E, Menashe S, Barrack RL, Bauer TW. Periprosthetic infection: what are the diagnostic challenges? J Bone Joint Surg Am. 2006;88 Suppl 4:138-47.

4. Ghanem E, Parvizi J, Clohisy J, Burnett S, Sharkey PF, Barrack R. Perioperative antibiotics should not be withheld in proven cases of periprosthetic infection. Clin Orthop Relat Res. 2007;461:44-7.

5. Bejon P, Berendt A, Atkins BL, Green N, Parry H, Masters S, McLardy-Smith P, Gundle R, Byren I. Two-stage revision for prosthetic joint infection: predictors of outcome and the role of reimplantation microbiology. J Antimicrob Chemother. 2010;65:569-75.

6. Choi HR, Kwon YM, Freiberg AA, Nelson SB, Malchau H. Periprosthetic joint infection with negative culture results: clinical characteristics and treatment outcome. J Arthroplasty. 2013;28:899-903.

7. Malekzadeh D, Osmon DR, Lahr BD, Hanssen AD, Berbari EF. Prior use of antimicrobial therapy is a risk factor for culture-negative prosthetic joint infection. Clin Orthop Relat Res. 2010;468:2039-45.

8. Parvizi J, Zmistowski B, Berbari EF, Bauer TW, Springer BD, Della Valle CJ, Garvin KL, Mont MA, Wongworawat MD, Zalavras CG. New definition for periprosthetic joint infection: from the Workgroup of the Musculoskeletal Infection Society. Clin Orthop Relat Res. 2011;469:2992-4.

9. Freeman MG, Fehring TK, Odum SM, Fehring K, Griffin WL, Mason JB. Functional advantage of articulating versus static spacers in 2-stage revision for total knee arthroplasty infection. J Arthroplasty. 2007;22:1116-21.

10. Huang R, Hu CC, Adeli B, Mortazavi J, Parvizi J. Culturenegative periprosthetic joint infection does not preclude infection control. Clin Orthop Relat Res. 2012;470:2717-23.
11. Kim YH, Kulkarni SS, Park JW, Kim JS, Oh HK, Rastogi D. Comparison of infection control rates and clinical outcomes in culture-positive and culture-negative infected total-knee arthroplasty. J Orthop. 2015;12(Suppl 1):S37-43.

12. Kim YH, Park JW, Kim JS, Kim DJ. The outcome of infected total knee arthroplasty: culture-positive versus culturenegative. Arch Orthop Trauma Surg. 2015;135:1459-67.

13. Parvizi J, Walinchus L, Adeli B. Molecular diagnostics in periprosthetic joint infection. Int J Artif Organs. 2011;34:847-55.

14. Cannon TA, Partridge DG, Boden RA, Townsend R, Stockley I. Case report of a successfully treated gentamicin and ciprofloxacin resistant Serratia marcescens prosthetic joint infection. Ann R Coll Surg Engl. 2014;96:e23-5.

15. Million M, Bellevegue L, Labussiere AS, Dekel M, Ferry T, Deroche P, Socolovschi C, Cammilleri S, Raoult D. Culturenegative prosthetic joint arthritis related to Coxiella burnetii. Am J Med. 2014;127:786.

16. Della Valle C, Parvizi J, Bauer TW, DiCesare PE, Evans RP, Segreti J, Spangehl M, Watters WC 3rd, Keith M, Turkelson CM, Wies JL, Sluka P, Hitchcock K; American Academy of Orthopaedic Surgeons. American Academy of Orthopaedic Surgeons clinical practice guideline on: the diagnosis of periprosthetic joint infections of the hip and knee. J Bone Joint Surg Am. 2011;93:1355-7.

17. Shahi A, Parvizi J, Kazarian GS, Higuera C, Frangiamore S, Bingham J, Beauchamp C, Valle CD, Deirmengian C. The alpha-defensin test for periprosthetic joint infections is not affected by prior antibiotic administration. Clin Orthop Relat Res. 2016;474:1610-5.

18. Melendez DP, Uhl JR, Greenwood-Quaintance KE, Hanssen AD, Sampath R, Patel R. Detection of prosthetic joint infection by use of PCR-electrospray ionization mass spectrometry applied to synovial fluid. J Clin Microbiol. 2014;52:22025.

19. Bereza PL, Ekiel A, Augusciak-Duma A, Aptekorz M, Wilk I, Kusz DJ, Wojciechowski P, Martirosian G. Identification of silent prosthetic joint infection: preliminary report of a prospective controlled study. Int Orthop. 2013;37:2037-43.

20. Deirmengian C, Kardos K, Kilmartin P, Cameron A, Schiller K, Parvizi J. Combined measurement of synovial fluid $\alpha$-Defensin and C-reactive protein levels: highly accurate for diagnosing periprosthetic joint infection. J Bone Joint Surg Am. 2014;96:1439-45.

21. Frangiamore SJ, Gajewski ND, Saleh A, Farias-Kovac M, Barsoum WK, Higuera CA. $a$-defensin accuracy to diagnose periprosthetic joint infection-best available test? J Arthro- 
plasty. 2016;31:456-60.

22. Achermann $Y$, Vogt M, Leunig M, Wust J, Trampuz A. Improved diagnosis of periprosthetic joint infection by multiplex PCR of sonication fluid from removed implants. J Clin Microbiol. 2010;48:1208-14.

23. Jacovides CL, Kreft R, Adeli B, Hozack B, Ehrlich GD, Parvizi J. Successful identification of pathogens by polymerase chain reaction (PCR)-based electron spray ionization timeof-flight mass spectrometry (ESI-TOF-MS) in culturenegative periprosthetic joint infection. J Bone Joint Surg Am. 2012;94:2247-54.

24. Jacob D, Sauer U, Housley R, Washington C, Sannes-Lowery K, Ecker DJ, Sampath R, Grunow R. Rapid and highthroughput detection of highly pathogenic bacteria by Ibis PLEX-ID technology. PLoS One. 2012;7:e39928.

25. Trampuz A, Piper KE, Jacobson MJ, Hanssen AD, Unni KK, Osmon DR, Mandrekar JN, Cockerill FR, Steckelberg JM, Greenleaf JF, Patel R. Sonication of removed hip and knee prostheses for diagnosis of infection. N Engl J Med. 2007;357:654-63.

26. Zimmerli W, Trampuz A, Ochsner PE. Prosthetic-joint infections. N Engl J Med. 2004;351:1645-54.

27. Cuckler JM. The infected total knee: management options. J Arthroplasty. 2005;20(4 Suppl 2):33-6.

28. Courtney PM, Melnic CM, Zimmer Z, Anari J, Lee GC. Addition of vancomycin to cefazolin prophylaxis is associated with acute kidney injury after primary joint arthroplasty. Clin Orthop Relat Res. 2015;473:2197-203.

29. Sewick A, Makani A, Wu C, O’Donnell J, Baldwin KD, Lee GC. Does dual antibiotic prophylaxis better prevent surgical site infections in total joint arthroplasty? Clin Orthop Relat Res. 2012;470:2702-7.

30. Parikh MS, Antony S. A comprehensive review of the diagnosis and management of prosthetic joint infections in the absence of positive cultures. J Infect Public Health. 2016;9:545-56.
31. Parvizi J, Erkocak OF, Della Valle CJ. Culture-negative periprosthetic joint infection. J Bone Joint Surg Am. 2014;96:430-6.

32. Byren I, Bejon P, Atkins BL, Angus B, Masters S, McLardySmith P, Gundle R, Berendt A. One hundred and twelve infected arthroplasties treated with 'DAIR' (debridement, antibiotics and implant retention): antibiotic duration and outcome. J Antimicrob Chemother. 2009;63:1264-71.

33. Sukeik M, Patel S, Haddad FS. Aggressive early debridement for treatment of acutely infected cemented total hip arthroplasty. Clin Orthop Relat Res. 2012;470:3164-70.

34. Hartman MB, Fehring TK, Jordan L, Norton HJ. Periprosthetic knee sepsis: the role of irrigation and debridement. Clin Orthop Relat Res. 1991;(273):113-8.

35. Koyonos L, Zmistowski B, Della Valle CJ, Parvizi J. Infection control rate of irrigation and debridement for periprosthetic joint infection. Clin Orthop Relat Res. 2011;469:3043-8.

36. Brandt CM, Sistrunk WW, Duffy MC, Hanssen AD, Steckelberg JM, Ilstrup DM, Osmon DR. Staphylococcus aureus prosthetic joint infection treated with debridement and prosthesis retention. Clin Infect Dis. 1997;24:914-9.

37. Romano CL, Gala L, Logoluso N, Romanò D, Drago L. Twostage revision of septic knee prosthesis with articulating knee spacers yields better infection eradication rate than onestage or two-stage revision with static spacers. Knee Surg Sports Traumatol Arthrosc. 2012;20:2445-53.

38. Mortazavi SM, Vegari D, Ho A, Zmistowski B, Parvizi J. Two-stage exchange arthroplasty for infected total knee arthroplasty: predictors of failure. Clin Orthop Relat Res. 2011;469:3049-54.

39. Alijanipour P, Adeli B, Hansen EN, Chen AF, Parvizi J. Intraoperative purulence is not reliable for diagnosing periprosthetic joint infection. J Arthroplasty. 2015;30:1403-6.

40. Parvizi J, Gehrke T, Chen AF. Proceedings of the international consensus on periprosthetic joint infection. Bone Joint J. 2013;95:1450-2. 